\title{
Controlling Bimetallic Nanostructures by the Microemulsion Method with Sub-nanometer Resolution using a Prediction Model
}

David Buceta $^{\dagger,}$, Concha Tojo" ${ }^{\prime}$, Miomir B. Vukmirovic ${ }^{\ddagger}$, F. Leonard Deepak ${ }^{\S}$, M. Arturo LópezQuintela $^{*}+$

† Laboratorio de Magnetismo y Nanotecnología, University of Santiago de Compostela, E-15782, Santiago de Compostela, Spain.

‡ Chemistry Department, Brookhaven National Laboratory, Upton, New York 11973, USA.

\| Physical Chemistry Department, University of Vigo, E-36310, Vigo, Spain .

${ }^{\S}$ International Iberian Nanotechnology Laboratory, Braga 4715-330, Portugal.

\section{Corresponding Author}

* M. Arturo López-Quintela (malopez.quintela@usc.es).

\section{ABSTRACT}

We present a theoretical model to predict the atomic structure of Au/Pt nanoparticles synthesized in microemulsions. Excellent concordance with the experimental results shows that the structure of the nanoparticles can be controlled at sub-nanometer resolution simply by changing the reactants concentration. The results of this study not only offer a better understanding of the complex mechanisms governing reactions in microemulsions, but open up a simple new way to synthesize bimetallic nanoparticles with ad-hoc controlled nanostructures.

KEYWORDS Microemulsion, sub-nanostructure, bimetallic, simulation, one-pot method. 


\section{Introduction}

Controlling the shape, size, structure, and composition of nanoparticles (NPs) is essential for various applications, ranging from plasmonics to catalysis ${ }^{1-3}$. Bimetallic nanoparticles (BNPs) are very complex due to the presence of an additional element. Adding an extra element does not only determine the material composition, but also enables the creation of different structures ${ }^{4}$. BNPs possess different properties from monometallic NPs, which were attributed to the electronic coupling between the individual metals in the system that is determined by composition and structure. For example, BNPs show significantly enhanced catalytic properties compared to monometallic NPs even at lower temperatures $^{5-6}$. Although there are several successful methods to prepare some of those structures, like the seeded-growth strategy to synthesize core-shell- and/or hetero-structured-NPs, the precise control of the bimetallic nanostructure using a one-pot technique remains a challenge ${ }^{7-10}$. A versatile method to control the size and composition of BNPs is the microemulsion route ${ }^{9-11}$. But even here, the high number of variables make it very difficult to tune the BNPs nanostructure, unless by changing the microemulsion system. It would be a great advance if the BNP nanostructure could be controlled with only one type of microemulsion, just by changing a single parameter, such as temperature or reactants concentration. Because many factors control the final structure of BNP, an enormous trial- and erroreffort would be required to achieve such a challenging objective. Hence, we developed a simulation model to predict the structure of BNPs in terms of type of microemulsion and metals employed. To test the model we compared model predictions with experimental data for a particular system. We chose $\mathrm{Au} / \mathrm{Pt} \mathrm{BNPs}$ first because it matches the model requirements but also because it shows high catalytic activity $^{7-8,12-14}$, which depends on the nanoparticle architecture. Specifically, controlling the surface composition is critical to improve the catalytic activity ${ }^{7}$. For example, a Pt shell on Au core is preferred for formic acid electrooxidation ${ }^{15}$, and oxygen reduction reaction ${ }^{16-17}$. On the contrary, Pt-Au alloyed shell is preferred for electro-oxidation of methanol ${ }^{18}$. 
Previous simulation studies on the synthesis of BNPs in microemulsions lead to two conclusions. First, the difference in the reduction rates of metal precursors determines the NP structure only if both reduction reactions take place at the same rate (a nano-alloy structure is invariably obtained), and if both reactions have very different rates (NP shows a core-shell structure). But the majority of BNPs of interest lies between these two extremes. For them, the NP structure strongly depends on the intermicellar exchange rate (the time needed for the droplets to collide and mix the reactants), mainly determined by the flexibility of the surfactant film ${ }^{19}$. A flexible surfactant allows a quicker exchange of material, favouring the mixture of both metals at atomic level. Secondly, the simulation model allowed to examine the influence of the nucleation rates of both metals on the final nanostructures and to assess how segregation of the metal is lowered by using higher concentrations of the metals precursors and/or surfactants with higher flexibility ${ }^{20}$. Based on these results ${ }^{19-20}$, we propose that the NPs structure could be modified by changing the local precursors concentration inside a droplet, and using a flexible surfactant film, which guarantees a more reach and labile behavior.

\section{Experimental}

Simulation model: Detailed simulation model can be consulted in Supporting Information.

Bimetallic nanoparticles preparation: Two 75\% Isooctane / 20\% Tergitol / 5\% water microemulsions (flexible film) were prepared, one containing the metal precursors $\left(\mathrm{HAuCl}_{4}\right.$ and $\left.\mathrm{H}_{2} \mathrm{PtCl}_{6}\right)$, and the other containing hydrazine (10 equivalents). The radius of the microemulsion droplets was $7.30 \mathrm{~nm}$ (calculated by DLS). Carbon black was added and stirred overnight and then microemulsion was broken with THF. Particles were washed with $\mathrm{EtOH}$ and $\mathrm{H}_{2} \mathrm{O}$ and dried prior to any further analysis. For Materials and more detailed experimental method see Supporting Information. 
HR-STEM and EDX characterization: Electron microscopy analysis was carried out with a FEI-TITAN $\mathrm{X}$-FEG transmission electron microscope used in STEM mode and operated at $300 \mathrm{kV}$. The images were acquired using a high angle annular dark field (HAADF) detector. The spherical aberration corrector (CEOS) for the electron probe, allowing an effective spatial resolution of $0.08 \mathrm{~nm}$. The column is also fitted with energy dispersive X-ray spectroscopy (EDS) which was employed for carrying out the compositional analysis. The $\mathrm{Au} / \mathrm{Pt}$ nanoparticles were prepared on a carbon coated $\mathrm{Cu}$ TEM grid.

\section{Results and discussion}

Simulations were carried out for different initial concentrations $\left(\left\langle\mathrm{c}\left[\mathrm{AuCl}_{4}\right]^{-}\right\rangle=\left\langle\mathrm{c}\left[\mathrm{PtCl}_{6}\right]^{2-}\right\rangle=\langle\mathrm{c}\rangle=2,16\right.$, 32, 64 reactants per droplet, which corresponds to $0.01 \mathrm{M}, 0.08 \mathrm{M}, 0.16 \mathrm{M}$ and $0.40 \mathrm{M}$, respectively) using a $\mathrm{v}_{\mathrm{Au}} / \mathrm{v}_{\mathrm{Pt}}=10$ reduction rate ratio and a flexible surfactant film. The left column in Figure 1 shows the nanostructures obtained at each concentration. To describe the structure, the sequence of metals of each nanoparticle is stored, and divided into ten concentric layers, assuming a spherical arrangement. The dispersion and averaged composition (\%Au) are calculated layer-by-layer. The layer composition is color-coded: The increase in proportion of Au goes from blue (0\%-10\%) to red (90\%-100\%). 50\% of each metal is represented by grey. The histograms in Fig. 1 (left column) show the number of particles containing a given percentage of Au, monitored from the NP core (inner layer) to the shell (outer layer). In this way, histograms show how the average composition changes from the beginning of the synthesis to the end. The NPs structure is also represented by concentric spheres, whose thickness is proportional to the number of layers with a given composition, keeping the same color scheme.

To validate the simulation, all the studied conditions were reproduced experimentally using a w/o microemulsion method. The Au/Pt particles were characterized using HR-STEM and cross sections scanned with EDX analysis revealed their structure. To compare the experimental and simulated results, 
theoretical STEM profiles were calculated from the simulated structures, computing the quantity of each metal crossed by a beam of $2 \AA$ (approximate size of the EDX beam). Both profiles were normalized to 1 and experimental $\mathrm{X}$ axis was changed from nm to counts for a better comparison. The centre and right columns in Fig. 1 show the STEM profiles, theoretical and experimental, of the average particle for each concentration. Both profiles show that the outer layers are enriched in the slower reduction metal (Pt), while the metal that is reduced faster (Au) accumulates in the core. A deeper Pt profile is obtained at high concentrations. A good agreement between experimental and theoretical results was attained, upholding the validity of the simulation model to predict the atomic structure of BNPs. According to these results, this simple approach of varying the concentration inside micelles leads to different nanostructures, ranging from a core-shell one where core is pure Au while the shell is Au-Pt alloy (see histogram $\langle c\rangle=2$ in Fig. 1) to a typical core-shell with different degrees of metal segregation depending on concentration. The Au core covered by a mixed shell obtained at a low concentration evolves into a more mixed core covered by a pure Pt shell as concentration increases (see decreasing red bar on the left and increasing blue bar on the right in histograms of Fig. 1). What is more important is that the nanostructure can be fine-tuned with sub-nanometer resolution.

The different nanostructures obtained by changing concentration can be explained by the particular nature of the microemulsion as a nanoreactor. At the beginning of the synthesis, when the core is forming, the slow reaction rate of forming Pt atoms causes that the Pt salt accumulates inside the micelle because the reaction medium is compartmentalized. The accumulation of Au salt is negligible due to its fast reduction rate, so the intermicellar exchange is the controlling step. We propose that Pt accumulation favors the chemical reduction as in a cage effect ${ }^{21-22}$ : An increase in the local concentration of Pt salt causes a faster reaction rate, and, consequently, attenuates the difference in reaction rates of both metals. That is, Pt reduction is accelerated compared to that of Au by the increase in concentration. This deeply affects the structure nanoparticle’s core. Because the local accumulation 5 
of Pt salt is higher at higher concentration, the degree of mixture of both metals in the core will be more pronounced as concentration increases (see Fig. 1, from top to bottom). In spite of the earlier Pt deposition, high concentration also implies that the available Pt salt is higher, giving rise to the Pt shell. Summarizing, the structure of BNPs can be modified by changing only concentration: The core is enriched in the faster metal at low concentration, and the shell is enriched in the slower one at high concentration.
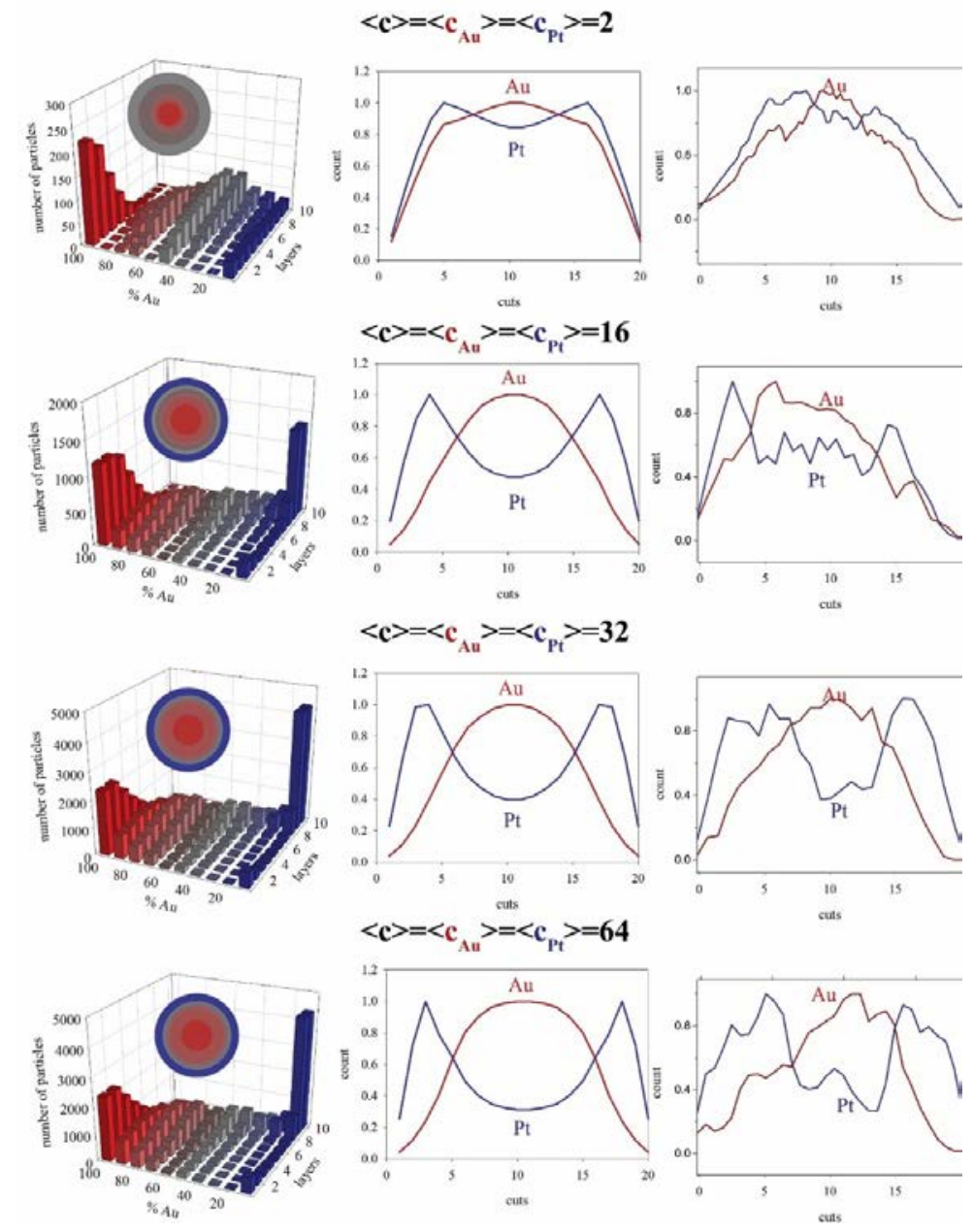

Figure 1. Left column: Theoretical histograms for different concentrations (Au:Pt $=1: 1)$. Colored spheres represent the average composition (red: Au, blue: Pt, grey: 50\% Au-Pt). As the color turns to lighter tonalities, the proportion of pure metal in the layer is higher. Centre column: Calculated STEM profiles for the average nanoparticle. Right column: Measured STEM profiles. The STEM images can be consulted in Supporting Information. 
To further explore the possibilities of this strategy, we carry out a second set of experiments fixing the total concentration $(\langle c\rangle=64$ reactants per droplet), but varying the ratio between them. For 75\%, 62.5\%, $50 \%, 37.5 \%$ and $25 \%$ of $\mathrm{Au}$, the particle structure was predicted theoretically and analyzed experimentally, as shown in Figure 2. As content of Au increases, the Pt shell disappears. The thickness of the pure Pt shell declines from 25\% Au until 50\% Au. With a further increase of Au content, the outer layers show a slightly Pt enrichment (see Au 62.5\%) or an alloy (see Au 75\%).

As before, the formation of these different nanostructures is due to the impact of the compartmentalization of the reaction media, which is different depending on reduction rates. Pt reduction rate depends on intermicellar exchange rate (as the case of $\mathrm{Au}$ ), but in addition, depends on reactant accumulation. Thus, from the core-shell BNP obtained at 50\% $\mathrm{Au}$, an increase of Pt\% leads to more Pt in the core (due to cage effect) covered by a thicker Pt shell. In contrast, Pt is delayed to outer layers at higher $\mathrm{Au} \%$. 

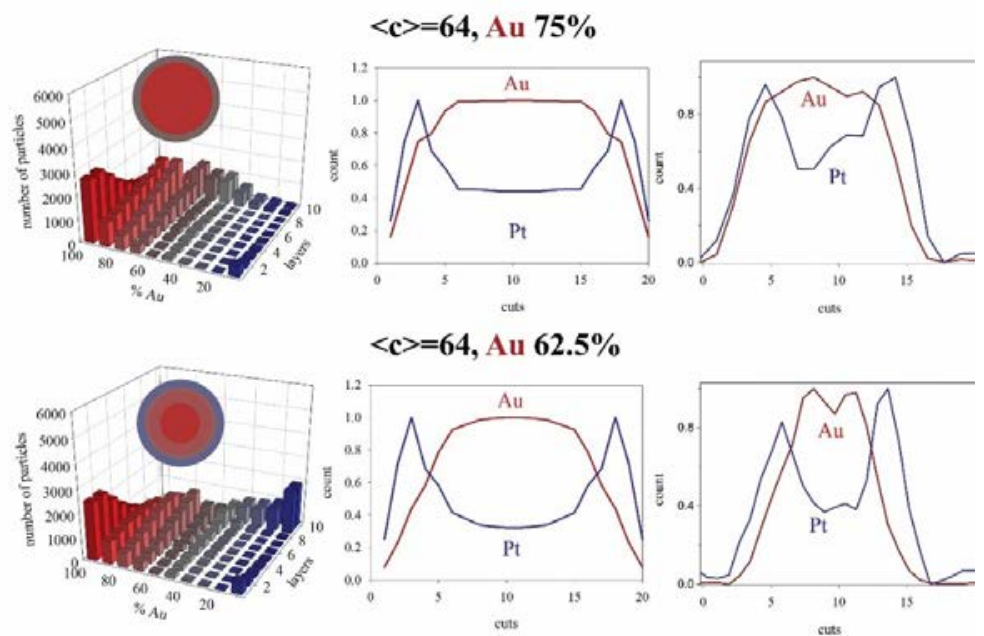

$<\mathrm{c}>=64, \mathrm{Au} 62.5 \%$
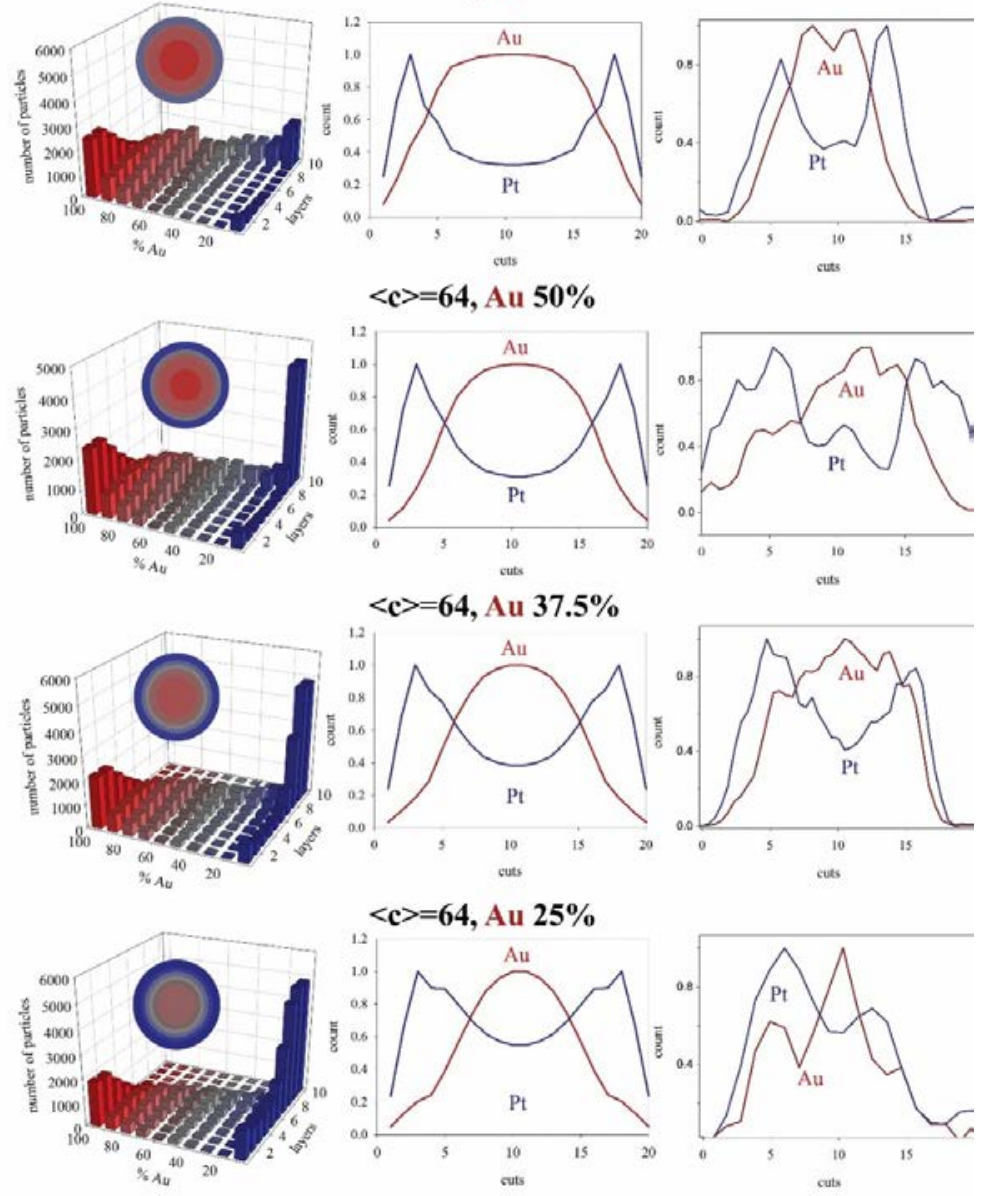

Figure 2. Left column: Predicted histograms for different initial Au:Pt ratios keeping fixed concentration $\langle c\rangle=64$. Colored spheres represent the averaged nanoparticle structure (red: Au, blue: Pt, grey: $50 \% \mathrm{Au}-\mathrm{Pt}$ ). As the color turns to lighter tonalities, the proportion of pure metal in the layer is higher. Centre column: Calculated STEM profiles. Right column: Measured STEM profiles. The STEM images can be consulted in Supporting Information.

\section{Outlook}

We present results obtained with a simulation of a one-step microemulsion process that generate $\mathrm{Au} / \mathrm{Pt}$ bimetallic NPs. Au/Pt bimetallic NPs were synthesized using the same conditions than those of simulation studies and the structure was determined experimentally. Good agreement between the 
theoretical and experimental STEM profiles confirms the validity of the model, showing that the modulation of the nanoparticle structure can be attained with subnanometer resolution simply by changing the concentration or reactants proportion. These results are valuable not only to better understand the complex mechanisms governing the reactions in microemulsions, but in opening a simple new way to synthesize BNPs with ad-hoc controlled sub-nanostructures.

\section{AUTHOR INFORMATION}

Corresponding Author.* M. Arturo López-Quintela (malopez.quintela@usc.es).

Notes. The authors declare no competing financial interests.

\section{ACKNOWLEDGMENT}

Works at University of Santiago de Compostela and University of Vigo were supported by Ministerio de Ciencia e Innovación, Spain (MAT2012-36754-C02-01) and Xunta de Galicia (GRC2013-044, FEDER Funds, REDES 2014/019). Work at BNL was supported by the U.S. Department of Energy, Division of Chemical Science (Contract No. DE-AC02-98CH10886). We thank Dr. R. R. Adzic (BNL) for useful discussions.

Supporting Information: Materials, Bimetallic nanoparticles preparation and Detailed simulation model. This information is available free of charge via the Internet at http://pubs.acs.org/

\section{REFERENCES}

1. Ferrando, R.; Jellinek, J.; Johnston, R. L., Nanoalloys: From Theory to Applications of Alloy Clusters and Nanoparticles. Chem. Rev. 2008, 108, 845-910.

2. Sun, S.; Murray, C. B.; Weller, D.; Folks, L.; Moser, A., Monodisperse Fept Nanoparticles and Ferromagnetic Fept Nanocrystal Superlattices. Science 2000, 287, 1989-1992. 
3. Habrioux, A.; Vogel, W.; Guinel, M.; Guetaz, L.; Servat, K.; Kokoh, B.; Alonso-Vante, N., Structural and Electrochemical Studies of Au-Pt Nanoalloys. Phys. Chem. Chem. Phys. 2009, 11, 3573-3579.

4. Toshima, N.; Yonezawa, T., Bimetallic Nanoparticles - Novel Materials for Chemical and Physical Applications. New J. Chem. 1998, 22, 1179-1201.

5. Hernández-Fernández, P.; Rojas, S.; Ocón, P.; Gómez de la Fuente, J. L.; San Fabián, J.; Sanza, J.; Peña, M. A.; García-García, F. J.; Terreros, P.; Fierro, J. L. G., Influence of the Preparation Route of Bimetallic Pt-Au Nanoparticle Electrocatalyst for the Oxygen Reduction Reaction. J. Phys. Chem. B 2007, 111, 2913-2923.

6. Boutonnet, M.; Lögdberg, S.; Svensson, E. E., Recent Developments in the Aplication of Nanoparticles Prepared from W/O Microemulsions in Heterogeneous Catalysis. Curr. Opin. Colloid Interface Sci. 2008, 13, 270-286.

7. $\quad$ Suntivich, J., Xu, Z.; Carlton, C.E.; Kim, J.; Han, B.; Lee, S.W.; Bonnet, N.; Marzari, N.; Allard, L.F.; Gasteiger, H.A., et al. Surface Composition Tuning of Au-Pt Bimetallic Nanoparticles for Enhanced Carbon Monoxide and Methanol Electro-Oxidation. J. Am. Chem. Soc. 2013, 135, 7985-7991.

8. Chen, Y.; Wang, W. G.; Zhou, S., Size Effect of Au Seeds on Structure of Au-Pt Bimetallic Nanoparticles. Mater. Lett. 2011, 65, 2649-2651.

9. $\quad$ Parapat, R. Y.; Parwoto, V.; Schwarze, M.; Zhang, B.; Su, D.-S.; Schomäcker, R., A New Method to Synthesize Very Active and Stable Supported Metal Pt Catalysts: ThermoDestabilization of Microemulsions. J. Mater. Chem. 2012, 22, 11605-11614.

10. Yin, Z.; Ma, D.; Bao, X., Emulsion-Assisted Synthesis of Monodisperse Binary Metal Nanoparticles. Chem. Commun. 2010, 46, 1344-1346. 
11. Pileni, M. P., The Role of Soft Colloidal Templates in Controlling the Size and Shape of Inorganic Nanocrystals. Nature Mater. 2003, 2, 145-150.

12. Zhou, S.; Mcllwrath, K.; Jackson, G.; Eichhorn, B., Enhanced CO Tolerance for Hydrogen Activation in Au-Pt Dendritic Heteroaggregate Nanostructures. J. Am. Chem.Soc. 2006, 128, 1780-1781.

13. Luo, J.; Njoki, P. N.; Lin, Y.; Mott, D.; Wang, L.; Zhong, C.-J., Characterization of Carbon-Supported Aupt Nanoparticles for Electrocatalytic Methanol Oxidation Reaction. Langmuir 2006, 22, 2892-2898.

14. Zhang, W.; Li, L.; Du, Y.; Wang, X.; Yang, P., Gold/Platinum Bimetallic Core/Shell Nanoparticles Stabilized by a Fréchet-Type Dendrimer: Preparation and Catalytic Hydrogenations of Phenylaldehydes and Nitrobenzenes. Catal. Lett. 2009, 127, 429-436.

15. Zhang, G.-R.; Zhao, D.; Feng, Y.-Y.; Zhang, B.; Su, D. S.; Liu, G.; Xu, B.-Q., Catalytic Pt-on-Au Nanostructures: Why Pt Becomes More Active on Smaller Au Particles. ACS Nano 2012, 6, 2226-2236.

16. Hartl, K.; Mayrhofer, K. J. J.; Lopez, M.; Goia, D.; Arenz, M., AuPt Core-Shell Nanocatalysts with Bulk Pt Activity. Electrochem. Commun. 2010, 12, 1487-1489.

17. Shao, M.; Peles, A.; Shoemaker, K.; Gummalla, M.; Njoki, P. N.; Luo, J.; Zhong, C.-J., Enhanced Oxygen Reduction Activity of Platinum Monolayer on Gold Nanoparticles. J. Phys. Chem. Lett. 2011, 2, 67-72.

18. Zhao, L.; Thomas, J. P.; Heinig, N. F.; Abd-Ellah, M.; Wang, X.; Leung, K. T., Au-Pt Alloy Nanocatalysts for Electro-Oxidation of Methanol and Their Application for Fast-Response Non-Enzymatic Alcohol Sensing. J. Mater. Chem. C 2014, 2, 2707-2714. 
19. Tojo, C.; de Dios, M.; López-Quintela, M. A., On the Structure of Bimetallic Nanoparticles Synthesized in Microemulsions. J. Phys. Chem. C 2009, 113, 19145 -19154.

20. Barroso, F.; Tojo, C., Designing Bimetallic Nanoparticle Structures Prepared from Microemulsions. J. Phys. Chem. C 2013, 117, 17801-17813.

21. Atkins, P.; de Paula, J., Physical Chemistry; Oxford University Press, 2002; Vol. seventh edition.

22. Tojo, C.; de Dios, M.; Buceta, D.; López-Quintela, M. A., Cage-Like Effect in Au-Pt Nanoparticle Synthesis in Microemulsions: A Simulation Study. Phys. Chem. Chem. Phys. 2014, 16, 19720-19731. 
TOC GRAPHICS

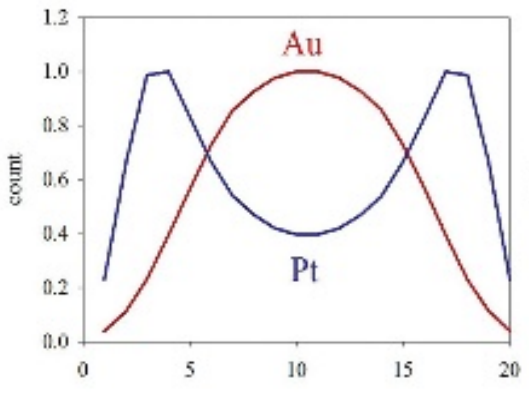

Theory

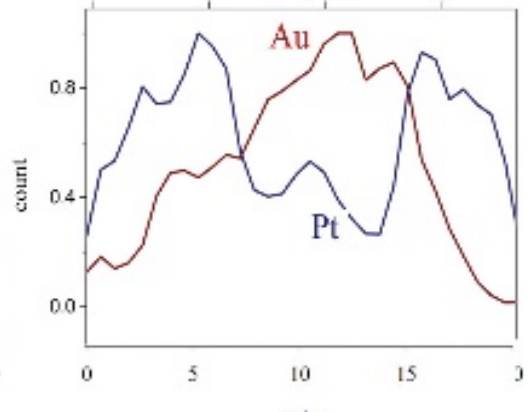

Experiment

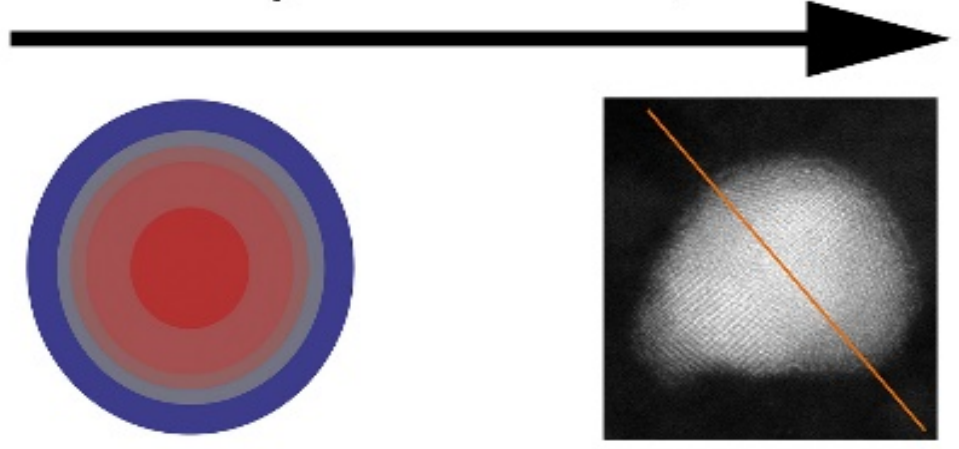

